\title{
ChemComm
}

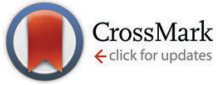

Cite this: Chem. Commun., 2016 52,1474

Received 9th November 2015, Accepted 30th November 2015

DOI: $10.1039 / \mathrm{c5cc09290f}$

www.rsc.org/chemcomm

\section{Extending human perception of electromagnetic radiation to the UV region through biologically inspired photochromic fuzzy logic (BIPFUL) systems $\uparrow$}

\author{
Pier Luigi Gentili, ${ }^{a}$ Amanda L. Rightler, ${ }^{\text {ab }}$ B. Mark Heron ${ }^{c}$ and \\ Christopher D. Gabbutt ${ }^{\mathrm{C}}$
}

\begin{abstract}
Photochromic fuzzy logic systems have been designed that extend human visual perception into the UV region. The systems are founded on a detailed knowledge of the activation wavelengths and quantum yields of a series of thermally reversible photochromic compounds. By appropriate matching of the photochromic behaviour unique colour signatures are generated in response differing UV activation frequencies.
\end{abstract}

A deep scientific understanding of how the human brain perceives, thinks, and acts will have a revolutionary impact in science, medicine, economic growth, security and well-being as recently expounded by an interdisciplinary and international team lead by Albus. ${ }^{1}$ Perception plays a relevant role within the cognitive architecture of the human nervous system. In fact, it builds and maintains an internal model of the external world and conditions behavior. $^{2}$ Among the different sensory systems, we have at our disposal, the visual system is remarkable because it allows us to discern colour, shape and the movement of objects. ${ }^{3}$ Human colour perception is founded upon a mosaic of many replicas of three types of photosensitive cells, termed cones. Each type of cone absorbs a particular region of the visible spectrum, although their spectra partly overlap. There is a type of cone that absorbs mainly blue light (with an absorbance peak at $420 \mathrm{~nm}$ ), another that absorbs green wavelengths (with an absorbance peak at $530 \mathrm{~nm}$ ), and a third type that absorbs up into the red region (with an absorbance peak at $565 \mathrm{~nm}$ ). When light, having a particular spectral power distribution, impinges on the retina, it activates each of the three types of cones by differing degrees. The distribution of

\footnotetext{
${ }^{a}$ Department of Chemistry, Biology and Biotechnology, University of Perugia, Via Elce di sotto 8, 06123 Perugia, Italy. E-mail: pierluigi.gentili@unipg.it

${ }^{b}$ Department of Chemistry and Biochemistry, University of Tulsa, Tulsa, Oklahoma, USA

${ }^{c}$ Department of Chemical and Biological Sciences, School of Applied Science, University of Huddersfield, Queensgate, Huddersfield, HD1 3DH, UK

$\dagger$ Electronic supplementary information (ESI) available: Instrumentation and materials and methods. The synthesis of 2-5 are described. The methodology for the determination of the photochemical quantum yields. Quantum mechanical simulations of the absorption spectra of the uncolored forms by the DFT method. The reflectance spectra of the photochromic sheets of white paper. See DOI: 10.1039/c5cc09290f
}

the degrees of activation of the three types of cones is information that travels as electrochemical signals up to the visual cortex. In the visual cortex, information is encoded as a specific pattern of activity of the cortical neurons in layer 4 of the V1 area. $^{4-7}$ Metameric matches ${ }^{8}$ occur when different spectral signals lead to the same activation patterns in the three types of cones and to the same pattern of activity in the visual cortex; thus, the different spectral signals appear to represent the same colour. Of course, the overall information about the colours within our brain is not limited to a simple correspondence between pigment activation and the spectrum of light. In fact, neuro-physiological evidence, such as colour constancy and coloured shadows, reveal the existence of post-receptor mechanisms for colour information processing. ${ }^{9,10}$ As far as the receptor mechanisms of colour perception are concerned, these mechanisms can be modelled by invoking the theory of fuzzy logic. ${ }^{11,12}$ Fuzzy logic is a mathematically rigorous model useful to describe the human computational ability which presently uses words and imprecise reasoning. ${ }^{13}$ It is based on the theory of fuzzy sets proposed by Zadeh 50 years ago. ${ }^{14} \mathrm{~A}$ fuzzy set is more than a "classical" set because it can not only wholly include or wholly exclude elements, but it can also partially include and exclude other elements. A fuzzy set breaks the law of excluded middle, because an element may belong to both a fuzzy set and its complement. The degree of membership $(\mu)$ of an element to a fuzzy set can be any real number included between 0 and 1. Fuzzy logic describes any non-linear complex input-output relations after building a fuzzy logic system. A fuzzy logic system consists of a collection of input fuzzy sets, a collection of output fuzzy sets, and a fuzzy inference engine that links, through syllogistic statements of the type "if. .., then...", each input fuzzy set to a particular output fuzzy set. The cones on the retina behave like input fuzzy sets: a beam of light impinging upon the retina belongs to the cellular fuzzy sets to different degrees, depending on its spectral power distribution. On the other hand, output fuzzy sets are made of clusters of patterns of activity of cortical neurons that are interpreted as the same colour. The fuzzy inference engine is the mechanism of transduction of the electrochemical information stored by the photoreceptor cells in the information encoded as patterns of activity of cortical neurons. 
This description is useful for the design of biologically inspired chemical systems for UV vision.

Humans cannot perceive UV radiation because the lens and cornea of the eye absorb strongly in this wavelength region preventing UV radiation from reaching the retina. However, a wide variety of animal species show sensitivity to UV, ranging from insects to mammals. Most often, the species that see UV are provided with a specific photoreceptor peaked around $350 \mathrm{~nm}$ in the UV-A region. ${ }^{15,16}$ UV sensitivity is useful in activities as diverse as navigation, intra- and inter-species communication, foraging and circadian synchronization. A remarkable case is the mantis shrimp that has at least four types of photoreceptors for UV in addition to eight for the visible region, as befits its habitat of kaleidoscopically colourful tropical coral reefs. ${ }^{17}$

In this work, we present the synthesis, study and optimized combinations of five thermally reversible photochromic compounds (1-5) that generate biologically inspired fuzzy logic systems useful to transform the frequencies of the UV spectrum, invisible to us, into specific colours perceptible to the human eye. The photochromic compounds are 1,3-dihydro-1,3,3-trimethyl-8'-nitro-spiro[2 $\mathrm{H}$-indole2,3'-[3H]naphth[2,1- $b][1,4]$ oxazine] (1), 2,2-bis(4-methoxyphenyl)-5,6dimethyl-2H-naphtho[1,2-b]pyran (2), 6-morpholino-3-(4-morpholinophenyl)-3-phenyl-3 $H$-naphtho[2,1- $b]$ pyran (3), 3,3-diphenyl-6morpholino-3H-naphtho[2,1- $b]$ pyran (4), 3-(2-fluorophenyl)-3(4-pyrrolidinophenyl)-3H-naphtho[2,1-b]pyran (5). Their structures and the colours of their solutions containing their ring-opened forms after UV irradiation are shown in Fig. 1.

The absorption spectra of the closed uncoloured forms are depicted in Fig. 2A. Naphthoxazine 1 has the largest absorption coefficient in the UV-A (320-400 nm) and almost in the entire UV-B region (280-320 nm). In the portion of UV-C included between 250 and $280 \mathrm{~nm}$ that will be considered in this work, the absorption of $\mathbf{1}$ is overwhelmed by the contributions of the other four naphthopyrans. Naphtho[2,1-b]pyrans 3 and 4 have fairly similar absorption spectra in the UV-A; in UV-B and UV-C regions, 3 absorbs more than $\mathbf{4}$ due to the presence of a morpholino group bound to one of the two phenyl rings (see Fig. 1). Naphtho$[1,2-b]$ pyran 2 is characterized by small values of the absorption coefficient in the range $387-294 \mathrm{~nm}$. Compound 5 is the naphtho[2,1- $b]$ pyran that commences absorption at the shortest

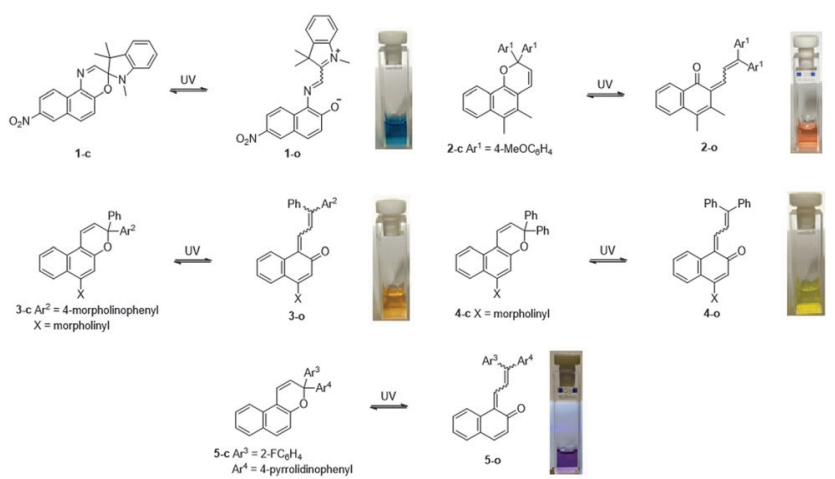

Fig. 1 Structures of the five closed uncoloured (1c-5c) photochromic compounds and their ring-opened coloured forms (10-50). The pictures show the colours they produce in MeCN solutions upon UV irradiation.

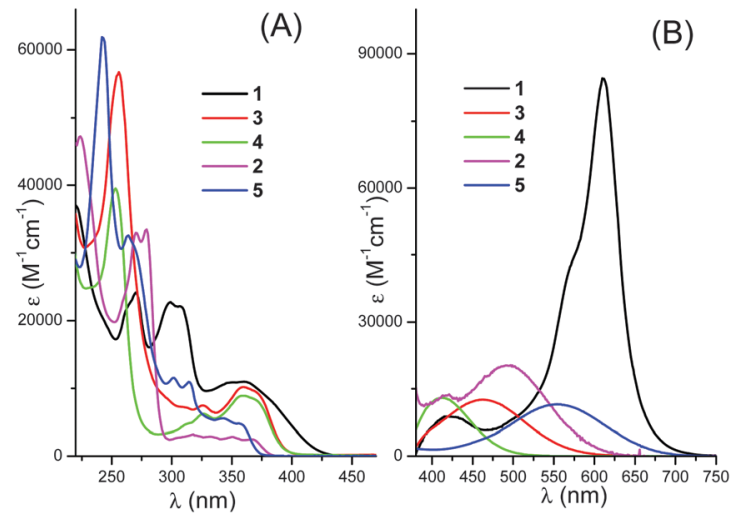

Fig. 2 Quantitative absorption spectra (recorded in $\mathrm{MeCN}$ ) of the closed uncoloured (A) and open coloured (B) forms of 1 (black traces), 3 (red traces), $\mathbf{4}$ (green traces), $\mathbf{2}$ (magenta traces), and $\mathbf{5}$ (blue traces).

wavelengths among the other photochromes. Upon UV irradiation (see Fig. 2B), 1 gives rise to a merocyanine that has an absorption band with a maximum at $610 \mathrm{~nm}$ and its solution becomes blue; 5 generates a band having a maximum at $554 \mathrm{~nm}$ and its solution becomes purple; 2 produces a band in the visible region which peaks at $497 \mathrm{~nm}$ and its solution becomes pink; 3 gives rise to a band centred at $463 \mathrm{~nm}$ and its solution becomes orange; finally, 4 generates a narrow band with a maximum at $413 \mathrm{~nm}$ and its solution appears yellow. The band due to $\mathbf{1}$ has an absorption coefficient at $610 \mathrm{~nm}$ that is more than four times larger than the values of the other coloured species; it is generally accepted that photochromic oxazines typically afford ring-opened species, which are more hyperchromic and bathochromic than those derived from diarylnaphthopyrans. ${ }^{18-21}$

The photochemical quantum yields $\left(\Phi_{\mathrm{PC}}\right)$ of the five photochromic compounds have been determined by irradiating at different wavelengths in the UV. The experimental methodology followed for their determination is described in the ESI. $\dagger$ The results are listed in Table 1 . For all compounds, $\Phi_{\mathrm{PC}}$ in the UV-C region is larger than $\Phi_{\mathrm{PC}}$ in UV-A and UV-B regions. This is particularly true in the case of naphthopyrans. In fact, naphthopyrans are known to give ultrafast electrocyclic ring opening reactions that kinetically compete with the other unreactive relaxation pathways and their photochemical quantum yields are usually wavelength-dependent. ${ }^{22}$ Simulations of the absorption spectra by density functional theory computation (DFT, see ESI $\dagger$ ) reveal that the electronic transitions in UV-A

Table 1 Photochemical quantum yields $\left(\Phi_{\mathrm{PC}}\right)$ determined by irradiating the five photochromic compounds in different regions of the UV spectrum, and lifetimes $(\tau)$ of their coloured forms (in MeCN solution)

\begin{tabular}{lllll}
\hline Compound & $\Phi_{\mathrm{PC}}(\mathrm{UV}-\mathrm{A})$ & $\Phi_{\mathrm{PC}}(\mathrm{UV}-\mathrm{B})$ & $\Phi_{\mathrm{PC}}(\mathrm{UV}-\mathrm{C})^{a}$ & $\tau=1 / k_{\Delta}(\mathrm{s})$ \\
\hline $\mathbf{1}$ & 0.12 & 0.07 & 0.18 & 25 \\
$\mathbf{2}$ & 0.17 & 0.26 & 0.45 & 37 \\
$\mathbf{3}$ & 0.24 & 0.25 & 0.92 & 37 \\
$\mathbf{4}$ & 0.27 & 0.42 & 0.53 & 61 \\
$\mathbf{5}$ & 0.28 & 0.23 & 0.62 & 19
\end{tabular}

${ }^{a}$ The quantum yields in the UV-C have been determined by irradiating with wavelengths longer than $250 \mathrm{~nm}$. 
and UV-B involve mainly the naphthopyran rings, whereas those in UV-C have charge transfer character from the naphthopyran rings to the two phenyl groups bound to the $\mathrm{sp}^{3}$ carbon atom in the pyran ring. The open forms produced by irradiating with UV have lifetimes of tens of seconds, which are independent of the frequency of irradiation (see data in Table 1). In particular, the open form of 5 has the shortest lifetime, lasting $19 \mathrm{~s}$, whereas the open form of $\mathbf{4}$ is approximately three times more persistent.

With knowledge of the spectral and photochemical properties of the five photochromes, some or all of them can be mixed in different ratios to create chemical systems able to transform the frequencies of the UV-A, UV-B and UV-C regions into different colours. The matching criteria are founded upon two considerations. First, the absorption bands of the uncoloured forms must be conceived as input fuzzy sets and the irradiation intensity $I_{0}\left(\lambda_{\text {irr }}\right)$ at $\lambda_{\text {irr }}$ will belong to each of them with a degree $\left(\mu_{\mathrm{Uv}, i}\right)$ given by:

$$
\mu_{\mathrm{UV}, i}=\Phi_{\mathrm{PC}, i}\left(\lambda_{\mathrm{irr}}\right) \cdot I_{\mathrm{abs}, i}\left(\lambda_{\mathrm{irr}}\right)
$$

In eqn $(1),{ }^{12,23} I_{\mathrm{abs}, i}\left(\lambda_{\text {irr }}\right)=I_{0}\left(\lambda_{\text {irr }}\right)\left(1-10^{-\varepsilon_{\mathrm{UV},}, l_{0, i}}\right)$ is the intensity absorbed by the uncoloured form of the $i$-th species whose absorption coefficient is $\varepsilon_{\mathrm{Un}, i}$ and whose analytical concentration is $C_{0, i}$. Second, the bands in the visible region produced by the open forms behave as output fuzzy sets. The contribution in absorbance of each coloured species at the wavelength $\lambda_{\text {an }}$ belonging to the visible region will be:

$$
A_{\mathrm{Co}, i}\left(\lambda_{\mathrm{an}}\right)=\frac{\varepsilon_{\mathrm{Co}, i}\left(\lambda_{\mathrm{an}}\right)}{k_{\Delta, i}} \mu_{\mathrm{UV}, i}
$$

where $k_{\Delta, i}$ is the reciprocal of the lifetime of the $i$-th open form. The final absorption spectrum recorded at the photo-stationary state will be the sum of as many terms represented by eqn (2) as there are photochromic components present in the mixture. Of course, the sum must be extended to all the wavelengths $\left(\lambda_{\mathrm{an}}\right)$ belonging to the visible spectrum.

Many combinations of photochromes 1-5, containing from three to five compounds and selected by applying eqn (1) and (2), have been found effective in distinguishing the three principal UV regions: UV-A (400-320 nm) from UV-B (320-280 nm) and from UV-C with $\lambda \geq 250 \mathrm{~nm}$. One of the best systems was a quaternary mixture involving 1, 4, 5, and 2 in concentrations of $5.2 \times 10^{-5} \mathrm{M}, 7.38 \times$ $10^{-5} \mathrm{M}, 1.4 \times 10^{-4} \mathrm{M}$, and $1.4 \times 10^{-4} \mathrm{M}$, respectively. Its discriminative power is shown in Fig. 3. When the system is irradiated by frequencies belonging to UV-A, the solution becomes green; under UV-B, the solution turns to grey, and under UV-C irradiation with wavelengths longer than $250 \mathrm{~nm}$, it becomes orange. The spectra recorded at the photo-stationary states, shown as the grey dashed traces in the bottom panels of Fig. 3, are accurately reproducible by summing the spectral contribution of each species, expressed by eqn (2) (see the red dashed traces in the same panels). Eqn (2) provides a powerful means to predict the observed colour when the mixture is irradiated by many UV frequencies, simultaneously. For example, in Fig. 4, the experimental (grey dashed traces) and predicted (red dashed traces) spectra obtained under different polychromatic irradiation frequencies are compared.

Under direct sunlight (see top-left panel), the solution becomes orange, and the experimental spectrum can be readily

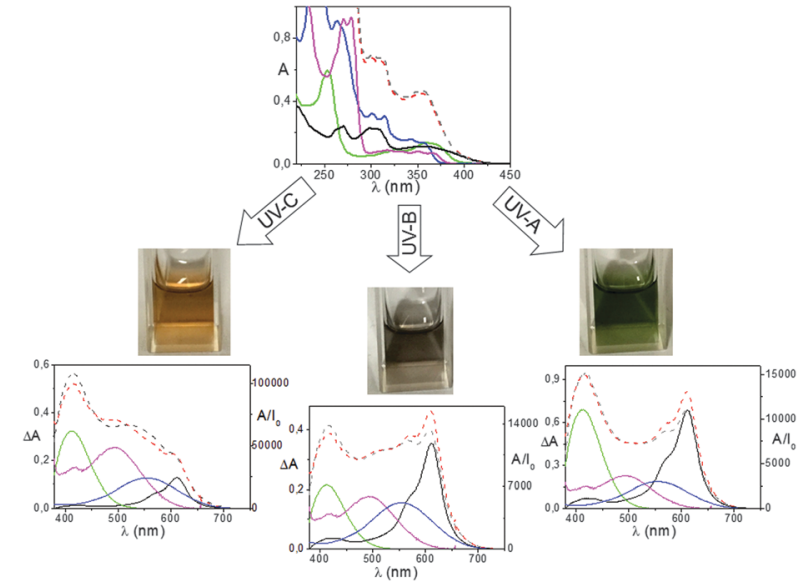

Fig. 3 Response of the quaternary photochromic fuzzy logic system to electromagnetic radiation belonging to the UV-A, UV-B and UV-C regions, respectively. Continuous traces represent the calculated spectral contribution of $\mathbf{1}$ (black), $\mathbf{4}$ (green), $\mathbf{2}$ (magenta), $\mathbf{5}$ (blue); their algebraic sum gives the red dashed traces. The grey dashed traces represent the spectra recorded experimentally using $125 \mathrm{~W}$ Xe lamp as irradiation source. In the three plots, $\Delta A$ is obtained by subtracting the spectrum recorded at the photo-stationary state from the initial one; $\left(A / I_{0}\right)$ represents the total absorbance into the visible calculated by eqn (2), divided by the total intensity $\left(I_{0}\right)$ at $\lambda_{\text {irr. }}$.

reproduced if we consider that the closed forms of $\mathbf{1}$ and $\mathbf{4}$ are completely transformed into their open forms by UV-A, whereas $\mathbf{2}$ and $\mathbf{5}$ are completely converted into their coloured forms by UV-B. When we add the contribution of the radiation at $254 \mathrm{~nm}$, emitted by the $\mathrm{Hg}$ lamp (see plot B1 in Fig. 4), the solution turns red because of the higher $\Phi_{\mathrm{PC}}$ of 2 and 5 in the
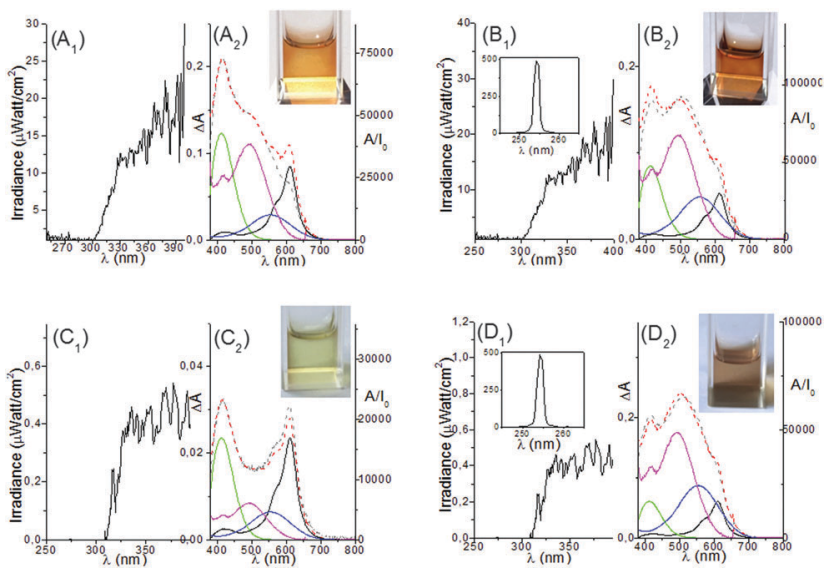

Fig. 4 Response of the quaternary photochromic fuzzy logic system to electromagnetic radiation belonging to different regions of UV. In each panel, the left-hand side plot represents the spectrum of the irradiation source, whereas the plot on the right shows the experimental spectrum (grey dashed trace) compared with the calculated spectrum (red dashed trace) obtained by summing the contributions of $\mathbf{1}$ (black continuous trace), $\mathbf{4}$ (green continuous trace), $\mathbf{2}$ (magenta continuous trace) and $\mathbf{5}$ (blue continuous trace). On the right-hand plot of each panel there is also a picture of the irradiated solution (inset). The top-left panel is relative to direct sunlight at 11 a.m.; the top-right panel is relative to direct sunlight at 11 a.m. plus irradiation at $254 \mathrm{~nm}$ emitted by a Hg lamp (inset); the bottomleft-hand panel is relative to skylight and the bottom-right panel is relative to skylight plus irradiation at $254 \mathrm{~nm}$ emitted by a Hg lamp (inset). 


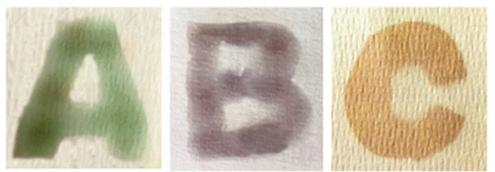

Fig. 5 Images of three sheets of white filter paper impregnated with optimal photochromic dye mixture and irradiated by UV-A (A), UV-B (B) (emitted by a $125 \mathrm{~W}$ Xe lamp) and UV-C (C) (emitted by a Hg lamp) radiation through negative masks reproducing the letters ( $A, B$ and $C$ ), respectively.

UV-C region. When the solution is irradiated by skylight but not direct sunlight, it assumes a pale green colour because the spectrum of skylight is deficient in UV-B (see plot C1 in Fig. 4). When we add the $254 \mathrm{~nm}$ wavelength emitted by the $\mathrm{Hg}$ lamp to the spectrum of skylight, the colour of the solution turns red because the very intense UV-C radiation quantitatively transforms the closed forms of $\mathbf{2}$ and $\mathbf{5}$ to their respective open structures. In fact, 2 and 5 have higher probabilities of absorbing the $254 \mathrm{~nm}$ radiation because they are at higher concentration in the mixture.

The performance of the Biologically Inspired Photochromic Fuzzy Logic (BIPFUL) systems investigated in acetonitrile solutions can be extended to a solid cellulose support as in white paper. After soaking a sheet of filter paper in an acetonitrile solution of the quaternary mixture described above, and after drying, the impregnated paper becomes photochromic and UV selective. It is possible to write on the paper by using UV radiation, and it is possible to change colour by appropriate tuning of the frequency of the irradiation source. For example, Fig. 5 shows a green A, a grey $\mathrm{B}$ and an orange $\mathrm{C}$ written on sheets of paper by irradiating them through negative masks and with radiation belonging to UV-A, UV-B or UV-C, respectively. If the UV source is turned off, the letters slowly (in roughly 30 minutes) disappear.

This work has demonstrated for the first time that solutions of carefully matched thermoreversible photochromic compounds are chemical systems having the emergent property of discriminating the three UV regions of the electromagnetic spectrum. These results contribute to the development of Systems Chemistry ${ }^{24}$ and Chemical Artificial Intelligence. ${ }^{25,26}$ In fact, the BIPFUL systems described herein mimic the computing power of vertebrates and invertebrates that have different photoreceptors to distinguish between frequencies of the electromagnetic spectrum. Recently, de Silva et al., ${ }^{27}$ demonstrated that the parallel processing by the combination of a $\mathrm{pH}$ sensor and a photo-acid generator detects the edges of objects, which is a rather complex computational task normally requiring a highly organized biomolecular system. This paper constitutes a further demonstration of how simple molecular logic systems, such as the photochromic compounds defined as "optical transistors", ${ }^{28-31}$ can give rise to high-level computing performances. We have shown that our BIPFUL systems can work not only in solution but also on a solid cellulosic support such as a sheet of inexpensive filter paper. Therefore, these photochromic systems are promising for designing new devices distinguishing UV frequencies in a photochemical manner instead of photo-electrically as with the use of semiconductors. ${ }^{32}$ Such photochemical UV detectors can be directly linked to human vision through the colours they produce and can supplement the performance of our visual system, enabling a further degree of detection and discrimination.

P. L. Gentili acknowledges the financial support by the University of Perugia (Fondo Ricerca di Base 2014, D. D. n. 170, 23/12/ 2014). A. L. Rightler thanks the American Chemical Society IREU Program and the National Science Foundation for support under award number DMR-1262908. The EPSRC are thanked for provision of a mass spectrometry service at the University of Swansea.

\section{Notes and references}

1 J. S. Albus, G. A. Bekey, J. H. Holland, N. G. Kanwisher, J. L. Krichmar, M. Mishkin, D. S. Modha, M. E. Raichle, G. M. Shepherd and G. Tononi, Science, 2007, 317, 1321.

2 J. S. Albus and A. Meystel, Engineering of Mind: An Introduction to the Science of Intelligent Systems, Wiley, New York, 2001.

3 C.-H. Sung and J.-Z. Chuang, J. Cell Biol., 2010, 190, 953.

4 B. R. Conway, Neuroscientist, 2009, 15(3), 274.

5 S. Zeki, Nature, 1980, 284, 412-418.

6 S. Zeki and L. Marini, Brain, 1998, 121, 1669.

7 P. Lennie and M. D'Zmura, Crit. Rev. Neurobiol., 1988, 3(4), 333.

8 K. R. Gegenfurtner, Nat. Neurosci., 2003, 4, 563.

9 S. G. Solomon and P. Lennie, Nat. Rev., 2007, 8, 276.

10 P. Brou, T. R. Sciascia, L. Linden and J. Y. Lettvin, Sci. Amer., 1986, 255(3), 84.

11 P. L. Gentili, Phys. Chem. Chem. Phys., 2011, 13, 20335.

12 P. L. Gentili, J. Intell. Fuzzy Syst., 2014, 27, 2137.

13 L. A. Zadeh, IEEE Comput. Intell. Mag., 2008, 3, 11.

14 L. A. Zadeh, Inform. Contr., 1965, 8, 338.

15 D. M. Hunt, S. E. Wilkie, J. K. Bowmaker and S. Poopalasundaram, Cell. Mol. Life Sci., 2001, 58, 1583.

16 M. J. Tovée, Trends Ecol. Evol., 1995, 10, 455.

$17 \mathrm{~J}$. Marshall and J. Oberwinkler, Nature, 1999, 401, 873.

18 V. Lokshin, A. Samat and A. V. Metelitsa, Russ. Chem. Rev., 2002, 71, 893.

19 J. D. Hepworth and B. M. Heron, in Functional Dyes, ed. S. -H. Kim, Elsevier, Amsterdam, 2006, ch. 3, pp. 85-135.

20 M. R. di Nunzio, P. L. Gentili, A. Romani and G. Favaro, ChemPhysChem, 2008, 9, 768.

21 S. Delbaere, G. Vermeersch and J.-C. Micheau, J. Photochem. Photobiol., $C, 2011,12,74$.

22 P. L. Gentili, A. Romani, R. S. Becker and G. Favaro, Chem. Phys., 2005, 309, 167.

23 M. Maafi, Molecules, 2008, 13(9), 2260.

24 R. F. Ludlow and S. Otto, Chem. Soc. Rev., 2008, 37, 101.

25 P. L. Gentili, RSC Adv., 2013, 3, 25523.

26 P. L. Gentili, Dyes Pigm., 2014, 110, 235.

27 J. Ling, G. Naren, J. Kelly, T. S. Moody and A. P. de Silva, J. Am. Chem. Soc., 2015, 137, 3763.

28 D. Gust, J. Andréasson, U. Pischel, T. A. Moore and A. L. Moore, Chem. Commun., 2012, 48, 1947.

29 K. Szaciłowski, Chem. Rev., 2008, 108, 3481.

30 Y. Wu, et al., Mater. Horiz., DOI: 10.1039/C5MH00223K.

31 W. Li, C. Jiao, X. Li, Y. Xie, K. Nakatani, H. Tian and W. Zhu, Angew. Chem., Int. Ed., 2014, 53, 4603.

32 E. Monroy, F. Omnes and F. Calle, Semicond. Sci. Technol., 2003, 18, R33. 